\title{
VOCABULARIO MARINERO DE COSTA RICA
}

\author{
Randi Korneliussen
}

\begin{abstract}
RESUMEN
El presente artículo describe y analiza el léxico del mar de acuerdo con el material recopilado en encuestas de carácter geolingüístico, realizadas en localidades situadas en las costas atlántica y pacífica de Costa Rica, en donde la principal fuente de trabajo es la pesca. Se incluye un glosario con los términos recopilados en el estudio geolingüístico.

Palabras clave: Léxico del mar, Costa Rica, glosario, análisis dialectológico.
\end{abstract}

\begin{abstract}
The present article describes and analyses the vocabulary used by fishermen and sailors in Costa Rica, such as tasks related to the sea and the act of fishing. The linguistic material was gathered along the Caribbean and Pacific coasts by means of a geolinguistic field work. In this article, a glossary of the recompiled vocabulary is included.

Key words: Terms of the Sea, Costa Rica, glossary, dialectological analysis.
\end{abstract}

\section{Introducción}

El presente trabajo es parte de una investigación geográfico-lingüística sobre el léxico marinero de las costas atlántica y pacífica de Costa Rica (ver los dos números anteriores de esta Revista). Esto abarca tanto el vocabulario usado para nombrar los fenómenos meteorológicos y las características geográficas de estas zonas, como el léxico relacionado con las actividades pesqueras y marineras. Los objetivos específicos son los siguientes:

Lic. Randi Korneliussen. Licenciada en Filología Española e Inglesa. Profesora en el Colegio Oystese Gymnas y Colegio Técnico de Norheimsund. Mediadora de conflictos para la región de Hordaland.

Correo electrónico: rankodyret@hotmail.com

Recepción: 20-5-04

Aceptación: 24-5-04 
1. Hacer una descripción lexicográfica del corpus.

2. Elaborar un glosario sobre marinerismos empleados en Costa Rica.

3. Describir, en la medida de lo posible, aspectos etnográficos y dialectales reflejados en el vocabulario.

En lo que concierne al léxico del mar, Costa Rica es campo virgen. Incluidas en las investigaciones lexicográficas de Quesada hay recopilaciones de palabras y expresiones marineras; sin embargo, hasta ahora no se ha desarrollado un estudio que trate exclusivamente el léxico marinero desde un punto de vista geolingüístico. Como ejemplos de trabajos sobre el léxico marinero que siguen la modalidad de Wörter und Sachen se pueden mencionar Chilenismos de Maintecillo. El lenguaje pesquero, de Stella Moder Pérez de Valenzuela (194749) y Terminología pesquera de la provincia de Valparaíso, de Elisa Carrasco (1956-57).

En cuanto al marco metodológico empleado en este trabajo, opté por seguir las pautas de estudios anteriores de esta índole e incluir en el glosario sólo las voces que no se daban en los diccionarios consultados, sobre todo el DRAE, las que se daban con otro significado, o como propias para Costa Rica u otras zonas latinoamericanas. Una ventaja de excluir voces comunes es que no se repite léxico ya elaborado y definido en otros diccionarios; pero tal sistema también tiene sus desventajas.

En este trabajo se presentaron efectos negativos imprevistos de antemano. A veces resultó muy difícil determinar si el significado que yo tenía para una voz correspondía con el del DRAE o con otros diccionarios consultados. Este era el caso sobre todo donde el significado dado en Costa Rica se mostraba menos amplio o general que en el diccionario. Un ejemplo es gancho 'uña del ancla'. El uso y la forma determinan los objetos cubiertos con esta palabra. La uña del ancla puede tener forma de gancho. Sin embargo, aunque el DRAE da varios ejemplos para gancho, el uso dado en Costa Rica no se incluye. En estas situaciones tuve que utilizar mi juicio para determinar si los significados dados eran compatibles. El problema más grave, sin embargo, es que el sistema de incluir sólo las voces propias de Costa Rica o Latinoamérica da una imagen errónea respecto de las voces que reúnen más significados. Por ejemplo, en el glosario del presente estudio, camarote se presenta con dos significados. El tercer significado, que es el más común, se tuvo que excluir, porque cubre el uso normal en español. Este sistema hace que se pierda información documentada durante el trabajo con el material, y las inexactitudes pueden hasta causar problemas para estudios posteriores y provocar conclusiones equivocadas en éstos. En fin; el hecho de incluir todas las voces ahorra trabajo y da un resultado más completo, por lo menos si el total del cuerpo coleccionado no es excesivamente grande.

Quiero llamar la atención a ciertas debilidades con los diccionarios consultados. El DRAE a veces incluye significados importantes de voces corrientes sólo como explicaciones para otras voces. Un ejemplo es cima como en cima de la ola. Encontré la palabra por casualidad como definición para cresta de la ola. El Diccionario de panameñismos (Revilla 1976) muestra graves faltas de orden alfabético. Los dos fenómenos mencionados son deficiencias evidentes que reducen el valor utilitario de los productos. Pienso, además, que es desventajoso cuando algunos diccionarios regionales y nacionales no incluyen la categoría gramatical del léxico, siendo este un rasgo significativo en la clasificación de una voz, y para determinar coincidencia lingüística entre diferentes áreas. 


\section{Glosario}

\subsection{Indicaciones de uso}

En el glosario que sigue se ha recopilado el vocabulario obtenido durante las encuestas. Sólo se han incluido las voces que se desvían del significado y uso estándar en español, y aquellas que en el Diccionario de la Lengua Española de la Real Academia Española (1992) y el Nuevo diccionario de costarriqueñismos se dan como americanismos o costarriqueñismos.

Los términos en mayúscula realzados con negrita son los lemas que encabezan la entrada.

Las palabras en mayúscula no realzadas son voces que se explican en otro sitio del glosario, y, donde se señala, sinónimos del lema.

Si una palabra es seguida por un nombre entre paréntesis, significa que sólo se dio en este sitio. En el siguiente ejemplo en cursiva se ve que la palabra explicada, fitin, se dio sólo en Cuajiniquil, y que la definición de sacavuelta también está incluida en este glosario.

FITIN m. (Cuaj.) Pequeño mecanismo (...) que funciona junto con la SACAVUELTA.

Si en un segmento hay una voz clave que se repite varias veces en diferentes combinaciones de vocablos, esta se representa con el signo [ ]. Si la voz clave de un segmento se da por primera vez en una locución, se separa de los otros elementos de la locución con el signo [ $>$ ]; AGUA > LLENA = AGUA LLENA. I SECA = AGUA SECA. Salvo en algunos casos donde la similtud semántica entre los vocablos tiene preferencia ante el orden alfabético, este se sigue dentro de los segmentos.

Si las palabras se clasifican según su similitud semántica, se señala con una coma o con $y$, y no con [ I ]; por ejemplo, ANZUELO $>$ CORRIENTE, $\sim$ JOTA y $\sim$ RECTO $=$ el mismo tipo de anzuelo.

En los casos donde varias voces de un segmento pertenecen al mismo grupo lingüístico, clases de palabras, locuciones, etc., la clasificación se anuncia sólo después de la primera.

La clasificación criollo (cr.) se usa sólo en la provincia de Limón.

$\mathrm{Si}$ en otros lugares aparecen voces en inglés, se da la designación inglés (ing.).

En los casos donde una voz puede pertenecer a más de una categoría lingüística, esto se señala con [ / ]; por ejemplo, CUERDEAR intr. / tr. Un signo de interrogación indica inseguridad sobre la categoría lingüística de un vocablo. Los ejemplos se marcan con letras en itálica.

\subsection{Abreviaturas empleadas}

$\begin{array}{ll}\text { adj. } & \text { adjetivo } \\ \text { adv. } & \text { adverbio } o \text { adverbial } \\ \text { Arreo } & \text { Calle del Arreo } \\ \text { Coco } & \text { Playa del Coco } \\ \text { Cortés } & \text { Ciudad Cortés } \\ \text { Coyote } & \text { San Francisco de Coyote } \\ \text { Cuaj. } & \text { Cuajiniquil } \\ \text { cr. } & \text { criollo } \\ \text { d. } & \text { diminutivo }\end{array}$




$\begin{array}{ll}\text { f. } & \text { sustantivo femenino } \\ \text { fig. } & \text { figurado } o \text { figurada } \\ \text { impers. } & \text { impersonal } \\ \text { ing. } & \text { inglés } \\ \text { intr. } & \text { verbo intransitivo } \\ \text { Jim. } & \text { Puerto Jiménez } \\ \text { loc. } & \text { locución } \\ \text { m. } & \text { sustantivo masculino } \\ \text { nom. } & \text { nominal } \\ \text { pl. } & \text { plural } \\ \text { P. Moreno } & \text { Puerto Moreno } \\ \text { prep. } & \text { preposición } \\ \text { pron. } & \text { pronombre } o \text { pronominal } \\ \text { Punt. } & \text { Puntarenas } \\ \text { P. Viejo } & \text { Puerto Viejo } \\ \text { refl. } & \text { verbo reflexivo } \\ \text { s. } & \text { sustantivo } \\ \text { Tam. } & \text { Tamarindo } \\ \text { tamb. } & \text { también } \\ \text { Tort. } & \text { Barra del Tortuguero } \\ \text { tr. } & \text { verbo transitivo } \\ \text { v. } & \text { verbo } o \text { verbal } \\ \text { V. } & \text { ver }\end{array}$

A

A LA DERIVA V. PESCAR A LA DERIVA.

ABIELA f. (P. Viejo) Especie de achicador.

ACUADRAR tr. (P. Moreno) Poner un ancla en cada lado del bote para mantenerlo estable cuando no está en uso.

ACHIQUE m. (Garza) Agujero para la salida del agua en las embarcaciones rápidas.

AGAINST (P. Viejo) 1. adv. Junto a. Cerca de. AGAINST the BEACH: Junto a la playa. 2. prep. Contra. AGAINST the BREEZE: Contra la brisa.

AGARRADERA f. (Limón) Estaquilla a la que se ata el remo.

AGUA > LLENA loc. nom. (P. Moreno) Marea alta. Tamb. MAREA . I SECA Marea baja. I QUE BRILLA (Tort.) Dícese del mar cuando brilla debido a la fosforescencia o por el reflejo en el agua de una fuente de luz, por ejemplo la luna.

ÁGUILA f. 1. Tipo de arpón cuya punta tiene la forma de una flecha. Se usa en agua salada para coger pescado grande. V. dibujo en el mapa 42 A. 2. (Cuaj.) La punta del arpón llamado ARBALETA. V. dibujo en el mapa 42 A. 4. (Punt.) ANCLA DE V. MARIPOSA. 
AJILAR intr. (Tort.) Inclinarse la embarcación.

\section{AL GARETE V. PESCAR AL GARETE}

ALETA. f. (Punt.) Parte ancha inferior del remo. Paleta.

ALISTAR tr. 1. (Cortés) Preparar algo para utilizarlo. ALISTAR la cuerda: Preparar la cuerda de pescar atándole el anzuelo. 2. (Limón) Reparar la red.

ANCLA DE CRUZ loc. nom. (Coco) Tipo de ancla para fondo de arena. Tiene dos barras; una pequeña que atraviesa otra más grande, a manera de una cruz. Por debajo tiene una uña transversal con forma de medialuna. V. dibujo en el mapa 27 A.

ANCLARSE refl. (Jim.) Anclar. Tirar el ancla al agua.

ANCOR s. cr. (P. Viejo) Ancla. Del inglés anchor. Heavy / Light ANCOR.

ANDAR EN VIAJE V. VIAJE.

ANIDADA f. (Jim.) La caja de la brújula.

ANZUELO $\mathrm{m}$. (P. Viejo) I $\sim$ A loc. nom. (Tort.) Tipo de anzuelo que tiene la cabeza aplastada. I CORRIENTE, JOTA y RECTO loc. nom. Tipo de anzuelo en forma de jota, con un solo garfio. Existen diferentes tamaños de anzuelo, que se miden por números, sobre todo entre el 1 y el 7 (el 1 siendo más grande que el 7). NB: El sistema de numeración no es igual para los diferentes tipos de anzuelos. I CON CABEZA REDONDA (P. Moreno) Tipo de anzuelo que tiene el ojo redondo, que lo distingue del $\sim$ CON CABEZA APLASTADA. I CON CURVA, CON CABEZA APLASTADA, $\sim$ COREANO $\sim$ CHINO y TAIWANÉS Anzuelo muy curvado que sirve para pescar tiburón y demás peces grandes. Existen diferentes tamaños que se miden por número. En El Coco se describe como un anzuelo cuyo garfio tiene varias lengüetas. Sin embargo, esta característica no se da por ejemplo en Puntarenas, donde el garfio del $\sim$ COREANO (etc.) es igual al $\sim$ CORRIENTE. I $\sim$ CURVO (Quepos) Tipo de anzuelo curvado, que posiblemente corresponde al $\sim$ CON CURVA. I $\sim$ DE PARGO, $\sim$ SENCILLO Tipo de anzuelo que se usa para pescar pargo. I JAPONÉS (Golfito) Tipo de anzuelo parecido al $\sim$ COREANO, pero menos grueso y de peor calidad. I $\sim \mathbf{O}$ (Tort.) Tipo de anzuelo con la cabeza redonda.

A PROFUNDIDAD loc. adv. (P. Viejo) V. PESCAR A FONDO.

ARADO m. (Coco) Tipo de ancla con forma de arado, para fondo de barro. V. dibujo en el mapa $27 \mathrm{~A}$.

ARBALETA $\mathrm{f}$. Tipo de arpón para pescado y langosta. El astil funciona como una pistola; tiene una punta afilada y de metal que se dispara contra la presa. Dibujo en el mapa $42 \mathrm{~A}$. V. ÁGUILA (3).

ARENUA f. (Cuaj.) Tipo de ancla. 
ARMARIO m. Compartimento para provisión de alimentos comestibles.

ARO m. (Coyote) Salabre. Poco usado.

ARPÓN ${ }^{1}>$ CUCHILLA loc. nom. (Limón), DE CUCHILLO (Coco), PARA MANATÍ (Tort.) Tipo de arpón muy parecido al llamado STRIKING LINE, que se usa sobre todo para coger manatíes y peces grandes. I DE PESCADO (Tort.) Tipo de arpón con tres (o más) pinchos, que se usa sobre todo para coger langosta y pescado. Tamb. HAWAIIANA. I NORMAL (Limón), PARA TORTUGA (Tort.) Arpón pequeño, de aproximadamente 1, 5 pulgar de tamaño, que se usa para coger tortuga. I PARA CAMARONES (Tort.) Arpón muy parecido al DE PESCADO, pero con menos lengüetas, que se usa para coger camarón. I PUNTIAGUDO (Limón) Tipo de arpón para pescado.

ARRECIFE m. 1. Acantilado. Alto de rocas en la playa. 2. (P. Viejo) Punta de tierra que entra en el mar.

ARRIESGAR tr. (P. Moreno) Espantar. Causar que alguien se vaya corriendo por miedo. También CORRER.

ASEGURADOR m. (Jim.) Palo con un gancho en uno de los extremos. Se usa para sacar peces grandes fuera del agua, por si acaso no aguanta el hilo. Bichero.

AVENTURA f. (Tort.) Acto de salir al mar y regresar dentro de varios días. V. VIAJE.

\section{B}

BAJAR intr. (Cuaj.) Navegar a favor del viento.

BAJIAR intr. (Cuaj.) Refluir el agua, como resultado de la marea menguante.

BAJO m. (Cuaj.) Banco de peces en el agua.

BALEAR tr. (Limón) Agarrar.

BALSAS f. pl. (P. Viejo) Flotadores de la red hechos de balsa. Boyas.

BANCO > ALTO loc. nom. (Limón) Montecillo de arena en el mar. I DE NUBE (Tort.), NUBLOSO (Limón) Banco de niebla.

BANDA f. 1. Acantilado. 2. (Cuaj.) Donde se unen el mar y la tierra. Playa u orilla. 3. (Tam.) Refuerzo externo en la embarcación, a la altura de la borda. 4. (Golfito) Refuerzo interno a la altura de la borda. V. BORDADENTRO.

BANDARREAL f. Refuerzo interno de la embarcación, a la altura de la borda. V. BORDADENTRO.

BANDEAR intr. (Cuaj.) Navegar junto a tierra. Ir cerca de la banda.

BARRA f. (Limón) Punta de tierra que entra en el mar. 
BASE f. (Jim.) La barra del ancla.

BEACH s. cr. (P. Viejo) Playa.

BIN f. ? m. (Punt.) Tabla móvil del fondo de una embarcación.

BITA f. 1. (Cuaj.) La parte superior de la roda. 2. (Golfito) Pieza que va por la parte interior de la roda. Contrarroda.

BOAT WITH ENGINE loc. cr. (P. Viejo) Embarcación de motor. La palabra en inglés: MOTORBOAT, también se usa.

BODEGUITA f. (Limón) Compartimento para provisión de alimentos comestibles. Bodega.

BOLSA DE ARRASTRE loc. nom. (Quepos) Bolsa o saco en que acaba la RED DE ARRASTRE.

BOMBA f. Instrumento que se usa para sacar el agua de los barcos. Existen diferentes tipos: I DE ACHIQUE loc. nom. (Arreo) I DE AGUA. (Tam.) I DE MANO (P. Moreno) I $\sim$ ELÉCTRICA.

BOMBILLO m. La luz de la panga. Bombilla.

BORDADENTRO f. (Coco) Refuerzo interno de la embarcación, a la altura de la borda. Tamb. BANDA, BANDARREAL, CONTRABORDA, CONTRAFUERZA y DOBLE PISO.

BOTAGUA f. Agujero para la salida del agua en las embarcaciones.

BOTE > DE HABILLO loc. nom. (P. Viejo) Bote hecho de esta madera. I EMPUJE (Limón) Remolcador pequeño.

BOW s. cr. ( P. Viejo) Parte delantera de la embarcación. Proa.

BOWLIN m. Tipo de NUDO FALSO que consiste en dar dos vueltas a un palo con un extremo de la cuerda o mecate. Tamb. CHANCHO, PATACHANCHO, PATA DE CHANCHO, PATA DE CHANGO, PATECHANCHO, PATO CHANCHO, y VALLESTRINQUI DOBLE.

BOYITAS f. pl. (Limón) Flotadores de la red.

BOYONES f. pl. (Limón) Flotadores de la red.

BRAKE s. ing. (Tam.) Tabla donde se apoya el motor fuera de borda. V. ESPEJO. La palabra existe en inglés, pero con el significado de 'freno'.

BRAZADA f. Medida de longitud que comprende desde el extremo de una mano a la otra, extirando los brazos; braza.

BREEZE s. cr. (P. Viejo) Brisa. To go in favour of the BREEZE: Ir a favor de la brisa. 
BRIDGE s. ing. (Punt.) Cabina de un bote. El significado en inglés es 'puente de mando'.

BRIGHT adj. cr. (P. Viejo) Utilizada para nombrar el tiempo, la palabra significa 'bueno' o 'claro'. It's a BRIGHT day: Es un día claro.

BUCEAR tr. (Limón) Pescar buceando. La palabra se puede utilizar transitivamente (admite un objeto), al contrario al uso normal en español. BUCEAR conchas.

C

CAJÓN DE LA PROVISIÓN loc. nom. (Golfito) Compartimento para provisión de alimentos comestibles.

CALABAZO m. (P. Viejo) Achicador hecho del fruto de la calabaza.

CALAFATE m. (Punt.) V. GALAFATEADOR.

CALARSE refl. (Punt.) Esconderse. Dícese p. ej. de los pescados que se asustan por un ruido.

CALM SEA loc. cr. (P. Viejo) Mar quieto.

CALMADO adj. Que está en calma. Mar CALMADO.

CALÓN m. Cada uno de los dos MECATES que van verticalmente por cada lado de la red para mantenerla extendida en el agua, desde abajo y por arriba.

CAMAGÜEY m. (Punt.) Parte delantera cuadrada de un bote, donde descansa la tripulación.

CAMAROTE m. 1. Cama donde duermen los marineros en el bote. 2. (P. Viejo) Compartimento para provisión de alimentos comestibles en el bote.

CAMBIO DE MAREAS loc. nom. (Coco) El repunte. Parte inicial de un ciclo de mareas.

CAMBUSA f. (Punt.) Compartimento para provisión de alimentos comestibles.

CANALETEAR intr. (P. Viejo) Remar con canalete.

CANTO m. (Cuaj.) Acantilado.

CAPTURA f. Cantidad de pesca capturada por una embarcación durante una jornada.

CARRUCHA f. (Limón) Carrete para cuerda de nailon.

CASCO m. (P. Moreno) Embarcación hecha de una sola pieza.

CASETA $\mathrm{f}$. Cuarto en botes de tamaño mediano, por ejemplo una lancha, donde vive y trabaja la tripulación en el mar, y del cual se maneja el buque.

CASQUILLETE $\mathrm{m}$. Parte delantera de una PANGA. 
CATCH s. cr. (P. Viejo) Cantidad de pesca capturada por una embarcación durante una jornada.

CAYUCO m. Bote pequeño de fibra o madera.

CEBA f. (Punt.) Alimentos que el pescador ofrece a los peces para atraerlos y cogerlos. Carnada. Cebo.

CELAJE m. (Cortés) Fosforescencia del mar.

CELOSA adj. V. SER LA EMBARCACIÓN CELOSA.

CINTA f. (Limón) Refuerzo interno a la altura de la borda de la embarcación. (La palabra normalmente se usa para denotar refuerzo externo.)

CIRIAL m. Fondo de piedras cerca de la superficie. Coral.

CIRIO m. (Punt.) Fondo de piedras cerca de la superficie. I DE ARENA. Montecillo de arena cerca de la superficie.

CLARAOYA f. (Punt.) Abertura en la cubierta del buque. Escotilla. La palabra probablemente viene de claraboya.

CLOUDY DAY loc. cr. (P. Viejo) Día nublado.

COBIJA f. (Punt.) Tipo de vela.

COLA DE GALLO loc. nom. (Punt.) Parte trasera cuadrada de la embarcación.

COLOCHO m. (P. Moreno) Rizo, bucle. Dícese por ejemplo de la cresta de la ola.

COMPLETO adj. El estado más alto del ciclo de marea. Mar COMPLETO.

CONCHAR intr. (Garza) Pescar conchas.

CONTRABORDA f. (Coyote) Refuerzo interno de la embarcación, a la altura de la borda. V. BORDADENTRO.

CONTRAFUERZA f. (Tort.) Refuerzo interno de la embarcación, a la altura de la borda o en la obra muerta. V. BORDADENTRO.

CONTRAVIENTO m. (P. Moreno) Viento de cara. Viento contrario.

CORAL m. (Cuaj.) Banco de arena.

CORDÓN m. 1. (P. Viejo) Parte lateral de la embarcación. Lado. 2. Refuerzo externo a la altura de la borda.

CORDONES CORRIENDO EN LÍNEA loc. nom. (Cortés) Tipo de corriente marina.

COREANA V. CUERDA COREANA . 


\section{COREANO V. ANZUELO COREANO.}

CORRER tr. Espantar los peces y causar que se vayan corriendo por miedo. CORRER los pescados.

CORRIENTE f. / adj. V. ANZUELO CORRIENTE. I DEL NIÑO loc. nom. Corriente marina que viene de Galápagos, 4 - 5 millas por hora. Trae plancton y calamares. I FOJA (Quepos) Corriente marina fría que pasa por Ecuador y Galápagos. Posiblemente idéntica a la DEL NIÑO.

CORTES m. pl. (Tam.) Acantilado.

COSTERO adv. (Tam.) Cerca de la costa. I IR Navegar junto a tierra.

CRESPADO adj. (P. Moreno) Dícese de la ola con cresta.

CRISTO m. (Cuaj.) Ancla. Posiblemente es una derivación de PEDRO por etimología popular. I LARGAR EL loc. v. Tirar el ancla al agua.

CRUZ V. ANCLA DE CRUZ.

CUCARACHA f. (Quepos) Tapón que va en un hueco del barco, el cual chupa agua para el enfriamiento del motor.

CUCHILLA f. 1. (P. Viejo) Parte ancha inferior del remo. Paleta. 2. (Limón) SOBREQUILLA. 3. Cada una de las uñas del ancla. 4. V. ARPÓN CUCHILLA.

CUCHILLO m. 1. CUCHILLA (1 y 3). 2. V. ARPÓN DE CUCHILLO.

CUERDA f. Hilo de pescar. I A loc. adv. (Golfito), A LA ( P. Moreno) Manera de pescar con cuerda. I COREANA loc. nom. Tipo de cuerda hecha con trenzas de nailon quemado. Material de los camaroneros. I TAIWANESA (P. Moreno) Tipo de cuerda que viene en diferentes grosores. Es corriente utilizar su medida en milímetros como nombre de la cuerda, por ejemplo LA 050 (delgada) o LA 060 (más gruesa).

CUERDEAR intr. / tr. (Cuaj.) Pescar con cuerda.

CULO DE LA RED loc. nom. (Punt.) Bolsa en que acaba la red barredera.

CURVA f. 1. Cada una de las piezas curvas atravesada sobre la quilla de la embarcación. Cuaderna. 2. (P. Viejo) Pieza que va por la parte interior de la roda. 3. (P. Viejo) Parte de la mar que entra en la tierra. Bahía.

CURVETE m. (P. Viejo) Anzuelo curvado, posiblemente igual que el COREANO. 


\section{CH}

CHACOTA f. Ola.

CHANCHO Tipo de NUDO FALSO. V. BOWLIN.

CHANCHOR m. (Quepos) Carrete grande para arrollar los mecates.

CHANGO Tipo de NUDO FALSO. V. BOWLIN.

CHAPATIER f. ? m. (Limón) Olas muy seguidas. CHIMPANTIER.

CHAPEN SEA loc. nom / cr. ( P. Viejo) Mar con olas muy seguidas. La expresión probablemente es una versión criolla del inglés sharpened sea: 'mar agitada', o una derivación de OLAS CHAPLIN.

CHAPLIN adj. (Tort.) Tipo de viento. I OLAS $\sim$ loc. nom. Olas muy seguidas que se producen debido al viento.

CHARCO m. (P. Moreno) Sobrenombre local para el Golfo de Nicoya. Está picado el CHARCO.

CHILILLO DE MECATE loc. nom. (Golfito) Cabo con que se atan las boyas.

CHIMPANTIER f. ? m. (Limón) Olas muy seguidas. CHAPATIER.

CHINCHA f. (Punt.) Estaquilla a la que se ata el remo.

CHINCHORREAR intr. (Limón) Pescar con el chinchorro.

CHINGA f. (Punt.) Tipo de embarcación cuadrada.

CHINGUEAR intr. / tr. Pescar con anzuelo lanzado desde el barco cuando va navegando.

CHINO V. ANZUELO CHINO.

CHIQUERO m. (P. Viejo) Achicador.

CHORREAR tr. REGAR la red al agua con el bote en marcha atrás.

CHUCERO m. (Cortés) Tipo de arpón. V. CHUZO.

CHUMACUA f. (Punt.) Estaquilla a la que se ata el remo.

CHUNCHE m. Objeto, cosa. En realidad, cualquier cosa puede llamarse CHUNCHE, cuando no se conozca su nombre o no se lo quiera expresar. En la jerga marinera la palabra se utiliza para nombrar las embarcaciones. El CHUNCHE se hundió.

CHUZA f. (P. Viejo) Tipo de arpón. V. CHUZO

CHUZO m. Tipo de arpón con muchos dientes, para río. Ver dibujo en el mapa 42 B. Este arpón también se conoce con los nombres de CHUCERO y CHUZA. 
D

DANFOR m. (Coco) Tipo de ancla. V. MARIPOSA.

DAR MOLA loc. v. (Coco) Tirar la red al agua.

DEEP adj. cr. (P. Viejo) Profundo.

DEKIN f. ? m. (P. Viejo) Tabla móvil del fondo de una embarcación.

DEPT s. cr. (P. Viejo) Profundidad.

DERIVA. V. PESCAR A LA DERIVA.

DESBALANCEAR v. pron. (P. Viejo) Inclinarse la embarcación.

DESGARRAR tr. Arar el fondo con el ancla.

DIABLO m. (Arreo) Tipo de arpón.

DOBLE > PASADA loc. nom. (Coco) Tipo de NUDO FALSO. I PISO (P. Moreno) Refuerzo interno de la embarcación, a la altura de la borda. V. BORDADENTRO. I VUELTA (Cortés) Tipo de nudo.

DRENAJE m. (Punt.) Refuerzo externo a la altura de la borda.

DRIFT v. cr. (P. Viejo) Estar a la deriva.

$\mathbf{E}$

ENCALLARSE refl. Encallar. Dar la embarcación en una piedra o arena y no poder continuar.

ENCANDECENCIA f. (Jim.) Fosforescencia del mar. Tamb. FÓSFORO y FLORESCENCIA.

ENCRESTADO adj. (Cuaj) Encrespado. Dícese de la ola con cresta.

ENFIBRAR tr. (Limón) Poner estopa y brea o masilla en las junturas de las tablas del bote, para que no entre el agua. GALAFATEAR.

ENGAÑADA f. (Cuaj.) V. ENGAÑADOR (2).

ENGAÑADOR m. 1. Anzuelo con dos ganchos. 2. Pez de metal, o de otra materia dura, que se usa en la pesca como cebo para atraer y coger los peces.

ENQUILLAR(SE) intr. / refl. (Golfito) Dar en una piedra o arena y no poder continuar.

ENRACHADO adj. (Punt.) Que viene a ráfagas. Viento ENRACHADO. 
ESCALERITA f. (Quepos) Escalera para subir a un barco. Tamb. ESCARELILLO.

ESCARELILLO m. (Quepos) Escalera para subir a un barco. Tamb. ESCALERITA.

ESCUELA DE PESCADO loc. nom. (Jim.) Banco o mancha de peces en el agua.

ESCUT m. Manga de red colocada en un aro de hierro. Salabre.

ESLOGAN s. cr. (Limón) Parte lateral de la embarcación. Lado.

ESPADILLA f. (P. Moreno) Remo.

ESPEJO m. Tabla donde se apoya el motor externo de la embarcación. Espejo de popa.

ESTE $m$. Viento del este. V. NORTE y VIENTO.

ESTELA f. (Jim.) Cresta de la ola.

ESTILLA f. (Limón) Tabla que se pone en la parte de la superficie del bote, de la punta hasta la mitad, o por todo el bote, para que esté nivelado. También se llama QUILLA.

$\mathbf{F}$

FALSAQUILLA f. 1. (Cuaj.) Pieza que va por la parte exterior de la quilla para protegerla.

2. (Punt.) Pieza que va de proa a popa por la parte interior de la roda. 3. Tabla móvil del fondo de una embarcación.

FIBERGLASS s. cr. (P. Viejo) Tabla donde se apoya el motor en botes de fibra.

FIERRO DE GALAFATEAR loc. nom. (Punt.) Instrumento de hierro que sirve para galafatear. Con un mazo el FIERRO se clava entre las junturas de las tablas del barco para abrir campo.

FIGA f. (Punt.) Tipo de arpón.

FILAMENTO $\mathrm{m}$. (Coco) Tipo de cuerda negra.

FISH v. cr. (P. Viejo) Pescar. Let's go fishing: Vamos a pescar.

FISHBANK s. cr. (P. Viejo) Banco de peces en el agua.

FISHFOUNDER s. cr. (Tort.) Tipo de ecosonda.

FITIN m. (Cuaj.) Pequeño mecanismo de metal que se pone al hilo de pescar para prevenir que este se enrede. Funciona junto con la SACAVUELTA.

FIX v. cr. (P. Viejo) Reparar.

FLORESCENCIA f. (Cuaj.) Fosforescencia del mar. Tamb. ENCANDECENCIA y FÓSFORO. 
FLORESCENTE adj. Fosforescente. Dícese del mar cuando la fosforescencia causa que brille por la noche. También FORESCENTE.

A FLOTE . V. PESCAR A FLOTE.

FOAM s. cr. ( P. Viejo) Espuma, cresta de la ola.

FONDEAR intr. (Coco) Pescar A FONDO.

FONDEARSE refl. (Coyote) Fondear. Tirar el ancla al agua.

FONDO m. Ancla o instrumento pesado que sirve para anclar.

FORESCENTE adj. (P. Moreno) Fosforescente. Dícese del agua cuando brilla por la noche debido a la fosforescencia del mar. También FLORESCENTE.

FÓSFORO m. (Golfito) Fosforescencia del mar. Tamb. ENCANDECENCIA y FLORESCENCIA.

FREEZER s. ing. (Punt.) Compartimento frío para provisión de alimentos comestibles, sobre todo en barcos grandes. Congelador.

FRENO m. (Limón) Tipo de MECATE o nailon que sirve para relingar.

FRINO DE GALAFATE loc. nom. (Quepos) Instrumento para GALAFATEAR.

FUEGO EN EL AGUA loc. nom. (Limón) V. AGUA QUE BRILLA.

G

GAJO m. (Coco) Embarcación podrida, rota.

GALAFATE m. (Cortés), GALAFATEADOR Instrumento para GALAFATEAR.

GALAFATEAR V. ENFIBRAR.

GALÓN m. Especie de achicador.

GALLO m. (Tort.) Parte delantera puntiaguda del barco. I COLA DE loc. nom. (Punt.) Parte trasera cuadrada de la embarcación. I RABO DE En una embarcación, refuerzo que va por el interior de la quilla, en la popa.

GANCHO m. 1. (Tort.) Estaquilla a la que se ata el remo. 2. Tipo de ancla.

GARDUMEN m. Banco o mancha de peces en el agua. Cardumen.

GARRETEAR intr. (Coyote) Arar el fondo con el ancla. Garrar.

GAZA f. Presilla hecha en el extremo de una cuerda para hacer un nudo corredizo. 
GAZAR tr. (P. Viejo) Amarrar el anzuelo a la cuerda con una GAZA.

GEMELAS f. pl. ( Cortés) Olas muy seguidas.

GO CLOSE TO THE REEF V. REEF.

GO UPON A REEF V. REEF.

GOLFO m. Bahía del río.

GOLPEA $\mathrm{f}$. (Tort.) Olas muy seguidas.

GONIL f. ? m. En la embarcación: refuerzo interno o externo de madera, a la altura de la borda.

GRADA f. Escalera para subir a un barco. Peldaño.

GUALETA f. (Golfito) Parte ancha inferior del remo. Paleta.

GUERREAR v. fig. (P. Moreno) Arar el fondo con el ancla cuando ésta no quiere pegar. Se llama así porque el ancla va "lucha" por asirse del fondo.

GUÍAS f. pl. (Quepos) Las estaquillas a las que se atan los remos.

$\mathbf{H}$

HACER > MAREA loc. v. Salir al mar y regresar el mismo día. También IR A MAREAR y MAREAR. I SALIDA (P. Moreno) Ir a pescar. También SALIR.

HAWAIIANA f. (P. Viejo) Tipo de arpón con tres (o más) pinchos, que se usa sobre todo para coger langosta y pescado. Tamb. ARPÓN DE PESCADO.

HONDO m. (Punt.) La profundidad del mar. Hondura. La palabra cambia de categoría gramatical, de adj. a sust. Probablemente, el mismo fenómeno ocurre también en Puerto Moreno, Quepos, C. Cortés y Golfito. En Golfito tamb. profundo posiblemente en uso sust. I TRABAJAR EN LO loc. v. (P. Moreno) Pescar con la red en la profundidad. PESCAR A FONDO.

HOOK s. cr. (P. Viejo) Cada una de las uñas del ancla.

HOYO m. Agujero, por ejemplo para el drenaje de los botes. La palabra también se puede aplicar a agujeros que se producen por accidente. Tamb. HUECO. I ANGULAR loc. nom. (Tort.) Argolla del ancla con forma angular, por la cual se amarra el mecate o la cadena.

HUECO m. Agujero, por ejemplo para el drenaje de los botes. La palabra también se puede aplicar a agujeros que se producen por accidente. Tamb. HOYO. 


\section{I}

IR > A CONTRA loc. v. Ir contra. Ir en contra. Vamos A CONTRA del viento. I A MAREAR V. MAREAR. I A VIAJE, DE VIAJE, PARA VIAJE Ir de pesca por varios días.

ISLA DE ARENA loc. nom. (P. Viejo) Montecillo de arena en el mar.

$\mathbf{J}$

JAPONÉS V. ANZUELO JAPONÉS.

JULIÁN m. (Punt.) Viento del norte.

$\mathbf{L}$

LANCEAR tr. (Cortés) Tirar la red al agua.

LANZADA f. (Punt.) Parte delantera puntiaguda de la embarcación.

LARGAR ANZUELO loc. v. (Punt.) Tirar el anzuelo al agua para pescar.

LARGUERO m. (Limón) Parte lateral de la embarcación. Lado.

LICORÁN f. ? m. (Limón) Corriente del mar que se mueve de abajo hacia arriba.

LÍNEA f. Cuerda de donde cuelgan varios anzuelos. Se usa frecuentemente con un WINCHER, sobre todo para coger peces grandes, como el tiburón. I CON PLOMOS loc. nom. (Tort.) Relinga. I DE MANO LÍNEA que no rodea por un WINCHER, sino que se usa para PESCAR A MANO (2).

LINEAR intr. (Cuaj.) TROLEAR con la LÍNEA.

$\mathbf{L L}$

LLENAR intr. (Cuaj.) Subir la marea.

M

MACHETAZO m. (P. Moreno) Parte lateral de la embarcación.

MANEGUETA $\mathrm{f}$. La argolla del ancla por la cual se amarra el mecate o la cadena.

MANGA f. 1. (P. Viejo) Empuñadura del remo o canalete. Mango. 2. (P. Viejo) Estaquilla a la que se ata el remo.

MANO f. (P. Viejo) Empuñadura del canalete. La MANO del canalete.

MANOFILAMENTO $\mathrm{m}$. Cuerda plástica. 
MANTA f. Tela de algodón, de la cual se hacen velas.

MÁQUINA f. (P. Moreno) El motor de la embarcación. I EN EL CENTRO Motor céntrico, de diesel. I FUERA DE BORDA Motor fuera de borda, de gasolina.

MAR > AFUERA (Tam.) loc. nom. Alta mar. I ALTA (Punt.) Mar elevada, con olas largas. I ALZADO (Limón) Mar picado. I BAJO (P. Viejo) Mar manso, sin olas grandes. I LLENO Dícese de la marea cuando está en su nivel más alto. Marea alta. I QUEMADO (P. Viejo) Dícese del mar cuando brilla debido a la fosforescencia o por el reflejo en el agua de una fuente de luz, por ejemplo la luna. Tamb. AGUA QUE BRILLA. I SECO (Coco) Marea baja. Tamb. AGUA SECA.

MARCASONDA f. (P. Viejo) Ecosonda.

MARCO m. (Cuaj.) V. CURVA.

MAREA > ADENTRO loc. nom. (Cortés) Reflujo. Retroceso de las olas de la costa. I AFUERA (Cortés) Flujo. Avance de las olas a la costa. I COMPLETA Dícese de la marea cuando está en su nivel más alto. Marea alta. La marea está COMPLETA. I CORRIDA (Cortés) Excursión al mar que no dura más que un día. I HONDA, LLENA V. $\sim$ COMPLETA. I MEDIA (Tort.), MEDIA $\sim$ Parte intermedia de un ciclo de mareas. No se especifica si es por menguante o creciente. La MAREA está a MEDIA. I PARADA DE (Garza) El repunte. Parte final de un ciclo de mareas. I PEQUEÑN (Jim.) La parte final de un ciclo de mareas, cuando el nivel del agua es el más bajo. Marea baja. Tamb. $\sim$ SECA o AGUA SECA. I SECA Marea baja. Tamb. AGUA SECA. I VACIANTE (Jim.) Reflujo. Retroceso de las olas de la costa.

MAREAR intr. Salir al mar y regresar el mismo día. Tamb. IR A MAREAR.

MARIPOSA $\mathrm{f}$. Tipo de ancla para fondo de arena, cuyas uñas son dos piezas de hierro con forma triangular que se cierran cuando toca fondo. V. dibujo en el mapa $27 \mathrm{~A}$. Tamb. RAYA, DANFOR y ANCLA DE ÁGUILA.

MECATE $\mathrm{m}$. Cuerda o hilo de materias naturales o sintéticas. I CHILILLO DE loc. nom. (Golfito) Cabo con que se atan las boyas. I DE RELINGA (Tam.) Hilo con que se une la red a la relinga. I SANSÓN (Coco) Mecate de nailon blanco con trenzas.

MECHA (DE PISO) f. / loc. nom. Conjunto de hilos trenzados que se utilizan para GALAFATEAR los botes.

MEDIA > BAJANTE, VACIANTE loc. adj. Parte intermedia de un ciclo de mareas, cuando está bajando el nivel del agua. I CRECIENTE Parte intermedia de un ciclo de mareas, cuando está subiendo el nivel del agua. I MAREA loc. nom. Parte intermedia de un ciclo de mareas. No se especifica si es por menguante o creciente. La MAREA está a MEDIA.

MENEAR intr. (Punt.) Inclinarse la embarcación. Se está MENEANDO mucho.

MESA f. Tabla donde se apoya el MOTOR INTERNO de la embarcación. 
MOCHOR m. (Quepos) Ola larga.

MONTÓN m. (Coyote) Cantidad de pesca capturada por una embarcación durante una jornada.

\section{MOTOR > ADENTRO, CÉNTRICO,$\sim$ DE CENTRO, $\sim$ ESTACIONADO,$\sim$ INTERNO}

loc. nom. Motor que está situado en el interior de la embarcacion, a diferencia del motor fuera de borda. Normalmente en lancha o embarcación más grande.

MOTORBOAT s. cr. (P. Viejo) Embarcación de motor.

MOVE v. cr. (P. Viejo) Moverse o inclinarse (la embarcación).

MUEBLE DE COCINA loc. nom. (Golfito) Compartimento para provisión de alimentos comestibles.

MUSTAD m. Tipo de ANZUELO CORRIENTE.

$\mathbf{N}$

NAILON m. Hilo de pescar. I HILO DE loc. nom. Se usa como material para MECATES, redes y como hilo de pescar.

NAP s. cr. (P. Viejo) Parte delantera puntiaguda del barco.

NEVERA f. (Limón) Abertura en la cubierta del buque. Escotilla.

NORTE $m$. Viento del norte. Se utiliza con artículo determinado o indeterminado. Sopla el NORTE. Está soplando un NORTE. V. VIENTO.

NUDO > CORRIENTE loc. nom. 1. Tipo de nudo. 2. Denominación común para cualquier nudo que se usa mucho. I $\sim$ CHINO (Punt.) Tipo de nudo. I $\sim$ DE BOTÓN (Tam.) Tipo de nudo que se utiliza en la pesca. I DE BUEY (Cuaj.) Tipo de nudo que sirve para empalmar hilos o mecates. I DE COQUEÑO, DE GUSANO (Garza) Tipo de nudo para amarrar o añadir la cuerda. I $\sim$ DE CORBATA (Quepos) Tipo de nudo que se utiliza en la pesca. I DEL DIABLO (Jim.) Tipo de nudo para mecate. I DE RESORTE Tipo de nudo que se utiliza en la pesca. I DE SOGA Tipo de nudo que se utiliza en la pesca. Apto tanto para mecate como para hilo de pescar. I FALSO Denominación común para cualquier tipo de nudo que se deshace con facilidad, por ser hecho con lazos, por ejemplo el RABO DE MONO. I GEMELO (Tam.) Tipo de nudo que se utiliza en la pesca. I JAPONÉS (Punt.) Tipo de nudo. I REINAL Tipo de nudo para amarrar el anzuelo al hilo de pescar. I TOPADO (Coyote) Tipo de nudo.

ÑATA f. (Garza) Proa de una embarcación con la parte delantera cuadrada. 


\section{$\mathbf{O}$}

OESTE m. Viento del oeste. V. NORTE.

OLA > CHAPLIN loc. nom. V. CHAPLIN. I DE VIENTO (P. Moreno) Viento a ráfagas. I PARA SURFEAR (P. Viejo) Ola larga.

OREJA f. Empuñadura del remo o canalete.

ORILLADO adv. (Cortés) Cerca de la orilla. I IR loc. v. Navegar junto a tierra.Tamb. IR COSTERO.

\section{$\mathbf{P}$}

PADDLE s. cr. (P. Viejo) Canalete.

PADDLING BOAT loc. cr. (P. Viejo) Bote para canalete, por ejemplo la canoa o el CAYUCO.

PADERÓN m. Paredón, acantilado.

PALETA f. 1. La parte ancha inferior del remo; pala de remo. 2. (Limón) Especie de uña del ancla.

PALILLO m. (Cuaj.) Palo para mantener extendida una red en el agua. V. TRANQUE.

PANETA f. Parte trasera de la embarcación.

PANGA f. Embarcación pequeña, descubierta, de madera o fibra, que se maneja con remos o con motor fuera de borda.

PANTANERA $\mathrm{f}$. PANGA con la parte delantera cuadrada, que se usa en los pantanos.

PAÑO m. (Quepos) Nombre humorístico para la red. Vamos a REGAR el PAÑO. Tamb. TRAPOS.

PARADA DE MAREA loc. nom. (Garza) El repunte. Parte final de un ciclo de mareas.

PARRILLA FALSA loc. nom. (Coco) Tabla móvil del fondo de una embarcación.

PATACHANCHO Tipo de NUDO FALSO. V. BOWLIN.

PATAS DEL REZÓN f. pl. (Cuaj.) Las uñas del rezón.

PATECHANCHO Tipo de NUDO FALSO. V. BOWLIN.

PATO CHANCHO Tipo de NUDO FALSO. V. BOWLIN.

PEDRO m. Sobrenombre para el ancla, por metonimia. PEDRO significa 'piedra', que anteriormente era el FONDO más usado, aunque esta función se ha disminuido a favor del ancla de hierro en la actualidad. Largar / echar el PEDRO; Tirar el ancla al agua. 
PEGAR intr. Soplar el viento. Está pegando el NORTE. I EN EL ANZUELO loc. v. (P. Moreno) Enganchar la carnada al anzuelo.

PEINE m. (Quepos) Parte delantera puntiaguda de la embarcación.

PESCA > ARTESANAL loc. nom. Se usa para denotar las maneras de pescar sin equipo técnico o industrial. Pesca rústica o tradicional. I DE ARRASTRE (Punt.) Pesca donde se usan redes barrederas. V. RED DE BARRER. I INDUSTRIAL Denota los modos de pescar que cuentan con equipo técnico avanzado. I MANUAL 1. (Quepos) Modo de pescar con la TARRAYA. 2. (Tam.) El hecho de PESCAR A MANO (1).

PESCAR > A FLOTE, FLOTADO loc. v. Manera de pescar que consiste en dejar flotar los instrumentos de pescar, por ejemplo el TRASMALLO o la LÍNEA, en la superficie o cerca de ella. A la red se le coloca boyas para que no hunda. I A FONDO Manera de pescar que consiste en poner los instrumentos de pescar, por ejemplo el TRASMALLO o la LÍNEA, cerca del fondo, colocándoles plomos para que no floten. A la red además se le colocan anclas. También $\sim$ A MANO y $~$ A PLAN. I A LA DERIVA Manera de pescar, dejándo los instrumentos de pescar, por ejemplo la red, la LÍNEA o la CUERDA, derivando en la corriente la red sin ancla. También AL GARETE, GARETEADO y $\sim$ CORRERIZA. I AL GARETE, $\sim$ GARETEADO V. $\sim$ A LA DERIVA. I $\sim$ AL SUPERFICIE (Quepos) V. A FLOTE. I A MANO 1. V. A FONDO. 2. Manera de pescar sólo con anzuelo o cuerda, sin el uso de redes u otro equipo. 3. (Coyote) Dícese cuando el pescador se queda en el sitio de pescar, vigilando los instrumentos. Con este significado de la expresión, pescar con LÍNEA, por ejemplo, no es A MANO, ya que el equipo se deja solo en el sitio. I A MEDIA AGUA (Punt.) Manera de pescar que consiste en dejar los instrumentos de pescar, por ejemplo el TRASMALLO o la LÍNEA, flotar en el nivel medio del agua. I A PLAN, A PLANO V. A FONDO. I $\sim$ A PROFUNDIDAD. V. A FONDO. I CORRERIZA V. A LA DERIVA. I FIJO (Cortés) Manera de pescar con la red, dejándola fija en un sitio, usando anclas. I FLOTADO V. A FLOTE I POR ARRASTRE (Quepos) Pescar con redes de arrastre. I IR MAR AFUERA (Tam.) Ir de pesca por varios días. I IR Y VENIR A (Jim.) Salir a pescar y volver el mismo día.

PIANGUAR intr. Pescar pianguas (especie de ostión).

PICADURO m. (P. Moreno) El hecho de picar los peces el cebo.

PICO m. Cada una de las uñas del ancla.

PICHINGA f. Recipiente de plástico, en realidad para conservar o transportar líquidos, pero que además sirve como flotador para la red.

PIN s. cr. (P. Viejo) Tapón para cerrar un agujero en el barco.

PINES s. cr. pl. (Tort.) Las estaquillas a las que se atan los remos.

PIQUE m. El hecho de picar los peces el cebo. Se quitó el PIQUE. 
PISO m. Tabla móvil del fondo de una embarcación.

PLANCHADO adj. (Cortés) Dícese del mar cuando está en calma.

PLANEROS m. pl. (Coyote) Plomos de la red. Se nombran así porque ayudan a mantener la red plana en el agua.

PLÁSTICO m. (P. Viejo) Achicador hecho de plástico.

PLATAFORMA f. (P. Viejo) Parte delantera del barco.

PLAYÓN m. (Limón) Isla pequeña en el mar.

PLOMADAS f. pl. Plomos de la red. El DRAE registra plomada como una palabra de cantidad, para denotar el conjunto de plomos de la red. Sin embargo, en Costa Rica plomada también puede ser cada uno de los plomos.

PLUGHOLE s. cr. (P. Viejo) Agujero para la salida del agua en las embarcaciones.

PLUMERO m. Tipo de anzuelo utilizado para TROLEAR.

POLYTELEANO m. (Coco) Cuerda plástica de un sólo hilo.

POOL s. cr. (P. Viejo) Parte del mar que entra en la tierra.

POPA f. (Coyote, P. Moreno, P. Viejo) Parte delantera de la embarcación. Proa. (El significado registrado en otras partes del país corresponde al DRAE: Parte trasera de la embarcación.)

PUENTE m. (P. Viejo) Escalera para subir a un barco.

PUNTA DE PLANCHA loc. nom. (Cortés) Punta de tierra que entra en el mar.

PUNTAL f. 1. Parte delantera puntiaguda de la embarcación. 2. (Coco) Pieza que va por la parte interior de la roda. Contrarroda.

PUÑO m. Empuñadura del remo.

PURA VIDA V. TIEMPO PURA VIDA.

\section{Q}

QUILLA f. Tabla que se pone en la parte de la superficie del bote, de la punta hasta la mitad, o por todo el bote, para que esté nivelado. Tamb. ESTILLA.

$\mathbf{R}$

RABO DE > GALLO loc. nom. En la embarcación: refuerzo que va por el interior de la quilla, en la popa. I MONO. Tipo de NUDO FALSO que se utiliza por ejemplo para amarrar el anzuelo al hilo de pescar, o para líneas taiwanesas. 
RAFAGADO adj. (Tort.) Que viene en ráfagas. Viento RAFAGADO.

RAFAGAZOS DE VIENTO loc. nom. (Cuaj.) Viento a ráfagas.

RAYA f. (Cuaj.) Tipo de ancla. V. MARIPOSA.

RAZÓN m. Tipo de ancla con cuatro o cinco uñas. Rezón. V. dibujo en el mapa 27 B.

RED $^{2}$ f. Se usa en particular para denotar el TRASMALLO. I DE ARRASTRE loc. nom. Camaronera (tipo de red que se utiliza para coger camarones). Consiste de una bolsa grande y dos cabos que la sostiene al barco. La bolsa arrastra el fondo del mar cuando el barco adelanta en la superficie. Se usa en la hondura, a diferencia de la $\sim$ DE ARRASTRE ARTESANAL, que es para poca profundidad. I DE ARRASTRE ARTESANAL (Coco) Chinchorro. Red barredera con aro en forma de cono que se utiliza para pescar en poca profundidad. La red se tira al agua de un barco o de la orilla. Se saca a la playa por hombres tirando de los dos lados al mismo tiempo. Así logran barrer el fondo, atrapando la captura dentro del cono. I DE BARRER Red barredera, por ejemplo el chinchorro. I DE CÍRCULO (Coco) Red boliche. Tipo de red igual o semejante a la $\sim$ DE ARRASTRE ARTESANAL, que se usa sobre todo para coger atún. I DE FLOTAR (P. Viejo) V. DE SUPERFICIE. I DE LANGOSTA (Tort.) Red de nailon que sirve para coger langostas. I $\sim$ DE PROFUNDIDAD TRASMALLO que se coloca en la profundidad al tener más plomos y anclas que flotadores. V. tamb. $\sim$ DE SUPERFICIE. I DE RASTRAR (P. Viejo) V. DE ARRASTRE ARTESANAL. I DE SUPERFICIE (P. Viejo) TRASMALLO que se coloca en la superficie, teniendo más flotadores que plomos. Se conoce tamb. bajo el nombre de $\sim$ DE FLOTAR. V. tamb. $\sim$ DE PROFUNDIDAD.

REDONDEZ f. (Punt.) Olas o remolinos que no trepan, causados por corrientes marinas.

REEF s. cr. 1. Fondo de piedras cerca de la superficie. 2. (P. Viejo) Montecillo de arena en el mar. I GO CLOSE TO THE loc. cr. Navegar cerca del ARRECIFE. I GO UPON A Dar en una piedra o arena y no poder continuar.

REGAR tr. Meter la red en el agua poco a poco desde un bote. REGAR LÍNEA. Vamos a REGAR el PAÑO.

REGLA f. (Cuaj.) Refuerzo externo e interno de la borda. Regala.

REMO > DE UNA MANO loc. nom. (Limón) Remo parecido al canalete, pero más pequeño. Se usa un solo remo, y sobre todo en los ríos, por ejemplo si el nivel del agua no es suficientemente profundo como para utilizar el motor fuera de borda. I DE DOS MANOS Remo que se usa en pares de dos, remando desde el centro del bote con un remo en cada lado. Más grande que el canalete.

REMOLQUE m. Remolcador.

REPUNTA f. Estado más alto o más bajo de la marea, cuando cambia. Repunte. I A BAJAR, BAJANTE, VACIANTE Estado más bajo de la marea. I A CRECER, CRECIENTE Estado más alto de la marea. 
RESACA f. (Coco) Oleaje pequeño y seguido.

REZONEAR intr. (Cuaj.) Arar el fondo con el rezón.

RIBE s. cr. (P. Viejo) V. CURVA.

ROCKY GROUND loc. cr. (P. Viejo) Fondo de piedras cerca de la superficie.

RODA f. (Cuaj.) Contrarroda. V. PUNTAL (2).

ROLA f. (P. Viejo) Cada uno de los troncos de madera o cilindros de metal que se usan para varar la embarcación fuera o dentro del agua. Tamb. ROLE.

ROLE m. V. ROLA.

ROMPEOLA m. (Quepos) Acantilado. Costa cortada verticalmente.

\section{$\mathbf{S}$}

SACAVUELTA $\mathrm{f}$. (Cuaj.) Pequeño mecanismo de metal que se pone a la cuerda de pescar para prevenir que esta se enrede. Consiste de un cilindro con un ojo en cada extremo donde se amarra la cuerda. Los ojos son movibles y dan vueltas sobre el eje cilíndrico, dependiente de la dirección que toma la cuerda. Funciona junto con el FITIN.

SALIR intr. Ir a pescar. SALIR de pesca. SALIR a pescar. I A VIAJE loc. v. Ir de pesca por varios días.

SANDBANK s. cr. (P. Viejo) Montecillo de arena en el mar.

SANDGROUND s. cr. (P. Viejo) Fondo de arena en el mar.

SAYAR intr. / tr. (Quepos) Ir a pescar.

SCULL s. cr. (P. Viejo) Estaquilla a la que se ata el remo.

SEABOTTOM s. cr. (P. Viejo) Fondo del mar.

SEAFIRE s. cr. (P. Viejo) La palabra significa 'fuego en el agua'. Se aplica al mar cuando brilla, por ejemplo debido a la fosforescencia o el reflejo de la luna.

SECAR intr. (Cuaj.) Bajar la marea.

SECO V. TRABAJAR EN LO SECO.

SEÍDO adj. (Coyote) Dícese del mar cuando está en calma.

SEPIRO m. (Quepos) Tipo de bote de motor.

SER LA EMBARCACIÓN CELOSA loc.v. (P. Moreno) Inclinarse la embarcación. Aquí se saca paralelos entre la inseguridad mental de una persona celosa y el desequilibrio físico del barco inclinado. LA EMBARCACIÓN ES CELOSA. 
SIDE s. cr. (P. Viejo) Parte lateral de la embarcación. Lado.

SINK v. cr. (P. Viejo) Irse a pique. Hundir.

SINKING SAND loc. cr. (P. Viejo) Terreno pantanoso de arena.

SOBREBORDA f. Refuerzo externo a la altura de la borda.

SOBREPISO m. (P. Moreno) Tabla móvil del fondo de una embarcación.

SOBREQUILLA f. (Cuaj.) Refuerzo externo de la embarcación, en la obra muerta. También VERDUGUETE.

SONDA f. En algunos sitios es sinónimo de ecosonda.

STAND s. cr. (P. Viejo) Parte trasera de la embarcación.

STRIKING LINE loc. cr. (P. Viejo) Tipo de arpón para tortuga. Funciona con una punta de hierro con varias lengüetas, que se dispara contra la presa. V. dibujos en el mapa $42 \mathrm{~A}$.

SUBIR intr. (Cuaj.) Navegar en contra del viento.

SUCUCHO m. (Coyote) Parte delantera de una embarcación, donde descansa la tripulación.

SUMAMI m. (Cortés) Ola larga.

SUR m. Viento del sur. V. NORTE.

SURFEAR V. OLA PARA SURFEAR.

SWAMP s. cr. (P. Viejo) Terreno pantanoso junto al mar.

$\mathbf{T}$

TABLA FALSA loc. nom. (Coco) Tabla móvil del fondo de una embarcación.

TAIWANÉS V. ANZUELO TAIWANÉS.

TAIWANESA V. CUERDA TAIWANESA.

TAJA MUERTA loc. nom. (Punt.) Refuerzo externo de la embarcación, en la obra muerta.

TAJAMAR m. (Tam.) Pieza que va por la parte interior de la roda.

TALCO SIMPLE CON RESINA loc. nom. (Limón) Refuerzo interno de la embarcación, a la altura de la borda. Tapa los agujeros.

TALÓN (Jim.) V. CALÓN.

TAMPSHAN s. cr. (P. Viejo) Tabla donde se apoya el motor. 
TANQUE m. (Quepos) Recipiente que se usa para coger conchas y otros moluscos.

TAPA f. 1. Abertura en la cubierta del buque. Escotilla. 2. (Coco) Refuerzo interno a la altura de la borda. 3. Tabla móvil del fondo de una embarcación.

TARIMA f. Tabla móvil con huequitos del fondo de una embarcación.

TARRAYA f. (Cuaj.) Atarraya, esparavel. Red redonda en forma de campana, con plomos colgando en los extremos. Se utiliza sobre todo para coger carnada. V. dibujo en el mapa $34 \mathrm{~A}$.

TARRAYAR intr. (Limón) Pescar con la TARRAYA. Tamb. se usa como adverbio: Pescar tarrayando.

TEAR s. cr. (P. Viejo) Rasgón en una cosa.

TECLER intr. (P. Viejo) Utilizar el tecle.

TEJER tr. Reparar una red.

TELÉFONO m. (Cuaj.) Cabo que une la red con las boyas de la superficie, y con el ancla del fondo.

TERRALILLO adj. (Punt.) Dícese del viento del oeste.

TERRENO SUAVE loc. nom. (Punt.) Terreno pantanoso.

TIDE s. cr. (P. Viejo) Marea. I HIGH loc. nom. Marea alta. I LOW Marea baja.

TIE THE HOOK ON THE LINE loc. cr. (P. Viejo) Amarrar el anzuelo a la cuerda.

TIEMPO PURA VIDA loc. nom. (Coco) Muy buen tiempo.

TILINTEAR tr. (P. Viejo) Tender la red.

TIME s. cr. (P. Viejo) Tiempo en el sentido de 'estado atmosférico'. I BAD loc. cr. Mal tiempo. I PRETTY Buen tiempo.

TIMERÁN m. (Coco) Tipo de velero. La palabra probablemente viene de trimerán, que es un barco parecido a una catamarán, pero con tres cascos.

TORNILLO m. (Cortés) Pieza que va por la parte interior de la roda.

TRABAJAR > EN LO HONDO loc. v. (P. Moreno) Pescar con la red en la profundidad. PESCAR A FONDO. I EN LO SECO (P. Moreno) Pescar con la red cerca de la superficie cuando la marea está baja.

TRAER LUCES loc. nom. (Quepos) Abertura en la cubierta del buque. Escotilla.

TRANCANIL m. (Coco) Agujero para la salida del agua en las embarcaciones. 
TRANCAS f. pl. (Tort.) Las uñas del ancla.

TRANQUE adv. (Cuaj.) Manera de pescar con la red que consiste en clavar dos o más postes o PALILLOS de aproximadamente un metro y medio de altura en un estero cuando la marea está baja. Entre los postes se extiende un trasmallo sujetado solamente por la parte inferior. La red se queda al nivel del agua, pero se extiende en la altura cuando sube la marea. Esta forma de pescar está en extinción.

TRAPOS fig. pl. (Arreo) Nombre humorístico para la red. Vamos a echar los TRAPOS al agua. Tamb. PAÑO.

TRASMALLAR intr. (Cuaj.) Pescar con el TRASMALlO. Tamb. se utiliza como adv: Pescar trasmallando.

TRASMALLO $\mathrm{m}$. Tipo de red rectangular, entre aproximadamente 4 y 10 metros de largura, hecho de hilo natural o una materia sintética, como nailon o plástico. V. dibujo en el mapa 34 A. La fineza del hueco de la malla determina su uso: El PARA CAMARÓN tiene una malla entre 3 y 6 pulgadas, mientras que la del $\sim$ PARA PESCADO es menos fina (entre 6 y 10). La definición del DRAE no corresponde con el trasmallo de Costa Rica. V. tamb. RED DE SUPERFICIE y RED DE PROFUNDIDAD.

TRASTES m. pl. (Arreo) Tipo de achicadores.

TREPAR intr. 1. (Quepos) Subir el agua a la costa por la pleamar. 2. tr. Tender la red.

TROLEAR intr. Pescar con anzuelo lanzado desde el barco cuando va navegando. TROLEAR con PLUMERO. TROLEAR con ENGAÑADOR.

TUCO m. (Punt.) Cada uno de los troncos de madera que se usan para apoyar los lados de un barco cuando este está en el seco, por ejemplo en la playa.

TÚNEL m. 1. (Punt.) Abertura en la cubierta del buque. Escotilla. 2. Tubo que va de proa a popa por la parte interior de la roda, y por donde pasa el eje que va a la propela.

TURN v. cr. (P. Viejo) Inclinarse (el bote). I OVER loc. cr. Volcar.

$\mathbf{U}$

URNA f. (Coco) Caja para guardar la comida en el bote.

V

VALLESTRINQUI DOBLE Tipo de NUDO FALSO. V. BOWLIN.

VARILLA $\mathrm{f}$. Palo que sobre todo anteriormente se usaba en los botes para avanzar en los ríos, clavándola en el fondo. I RECIA loc. nom. (Coco) La barra del ancla. 
VENTANITA f. (Punt.) Abertura en la cubierta del buque. Escotilla.

VERDUGUETE m. Refuerzo externo de la embarcación, en la obra muerta. Verduguillo. También SOBREQUILLA.

VIAJE m. Excursión al mar. I COMPLETO loc. nom. (Golfito), DE EXPLORACIÓN (Tort.) Excursión al mar que dura varios días. I REDONDO (Tort.) Excursión al mar que no dura más de un día. I ANDAR EN $\sim$ loc. v., IR A $\sim$, IR DE $\sim$, IR PARA y SALIR A $\sim$ Ir de pesca por varios días.

VIENTO > A POPA o DE POPA loc. nom. Viento que sopla hacia el mismo punto a que se dirige la embarcación. Viento en popa. I A PROA o $\sim$ EN PROA Viento de proa. El que sopla en dirección contraria a la que lleva el buque. I DE TIERRA (P. Viejo) El que viene de la tierra. Viento terral. I DE AFUERA (P. Viejo) El que sopla desde el mar hacia la tierra. I ESTE Viento del este. I NORTE Viento del norte. V. NORTE. I OESTE Viento del oeste. I OLA DE $\sim$ (P. Moreno) Viento a ráfagas. I RAFAGAZOS DE (Cuaj.) Viento a ráfagas. I $\sim$ SUR Viento del sur.

VIENTOSO adj. Dícese cuando hace viento fuerte. Ventoso.

VIRAR intr. Inclinarse la embarcación.

VIVEROS m. pl. (P. Moreno) Agujeros en un barco, probablemente para la entrada del agua, para mantener los pescados vivos.

\section{W}

WAVE s. cr. Ola. I BIG $\sim$ loc. cr. Ola grande. I BROKEN $\sim$ Cresta de la ola. I HIGH $\sim$ Ola alta.

WINCHER s. ing. Carrete grande, normalmente eléctrico, con MECATE o hilo, que se utiliza para jalar objetos pesados.

WIND s. cr. (P. Viejo) Viento. I BLOWING loc. cr. Soplar el viento. I COMING FROM THE BACK Viento que sopla desde atrás. I EAST $\sim$ Viento del este. I HARD $\sim$ Viento fuerte. I IN FAVOUR Viento a favor. Con el viento. I LAND $\sim$ Viento que sopla desde la tierra hacia el mar. I NORTH $\sim$ Viento del norte. I OUT $\sim$ Viento que sopla desde el mar hacia la tierra. I SOUTH $\sim$ Viento del sur. I WEST $\sim$ Viento del oeste.

\section{Notas}

1. Dibujos de los arpones se pueden apreciar en el mapa 42 (A y B)

2. Dibujos de las redes se pueden apreciar en mapa 34 (A y B) 


\section{Bibliografía}

Abellán, J. L. 1972. La Idea de América. Origen y evolución. Madrid: Ediciones Istmo.

Agüero, A. 1962. El español de América y Costa Rica. San José: Atenea.

1964. "El español en Costa Rica y su atlas lingüístico". Presente y futuro de la lengua española. Madrid, OFINES. Tomo 1, 135-152.

Akman, Haçi. Sin fecha. Kurdisk identitet. Sin editorial.

Alvar, Manuel. 1969. Estructuralismo, geografía lingüística y dialectología actual. Madrid: Editorial Gredos S. A.

(ed.). 1977. Terminología marinera del Mediterráneo. Comisión española del A.L.E., Madrid.

1993. Estudios Canarios. Tomo II. Islas Canarias: Colección Clavijo y Fajardo.

1975. Atlas linguiístico - etnográfico de las Islas Canarias. (2 volúmenes). Gran Canaria: Ediciones del Cabildo Insular.

Alvar, Manuel et al. 1979. Atlas lingüístico - etnográfico de Aragón, Navarra y Rioja. 12 tomos. Zaragoza: Diputación Provincial, Dep. de Geografía Lingüística, Institución Fernando el Católico.

Alvar, M. et al. 1963-64. Atlas lingüístico - etnográfico de Andalucía. (5 volúmenes). Granada: Publicaciones de la Universidad de Granada.

Alvar, Manuel y A. Quilis. 1984. Atlas lingüístico de Hispanoamérica. Cuestionario. Madrid: Instituto de Cooperación Iberoamericana.

Araya, G. (red.), C. Contreras, C. Wagner y M. Bernales. 1973. Atlas lingüístico-etnográfico del Sur de Chile. Valdivia: Instituto de Filología de la Universidad Austral de Chile y Editorial Andrés Bello.

Auer, Peter y Aldo di Luzio (eds.). 1988. Variation and Convergence. Studies in Social Dialectology. Berlin, New York: Walter de Gruyter.

Bescht, Werner et al. 1982. Dialektologie. Ein Handbuch zur deutschen und allgemeinene Dialektforschung. Erster Halbband. Berlin - New York, Walter de Gruyter.

Buesa, T. y L. Florez. 1981-82. Atlas lingüístico - etnográfico de Colombia. 6 tomos. Bogotá: Instituto Caro y Cuervo. 
Bugge, Hans Erik. 1996. El léxico de los animales domésticos en el Valle Central y las Zonas Noroeste y Norte de Costa Rica. Tesina de Hovedfag: Universidad de Bergen.

Butler, Cristopher. 1985. Statistics in Linguistics. Nueva York: Basil Blackwell Ltd.

Cáceres, O. M. y D. Palacios. 1980. El léxico del pescador en Puerto Armuelles. David: Universidad de Panamá, Facultad de Filosofía, Letras y Educación.

Castellón, H. A. 1939. Diccionario de Nicaragueñismos. Managua: Talleres Nacionales.

Chambers, J.K. y P. Trudgill. 1980. Dialectology. Cambridge: Cambridge University Press.

Collado, Jesús Antonio. 1978. Fundamentos de Lingüística General. Madrid: Editorial Gredos.

Coseriu, Eugenio. 1985. El hombre y su lenguaje. Madrid: Editorial Gredos.

Dickeman, Margaret. 1985. "Eliminación de la ambigüedad pronominal en el discurso del criollo limonense”. Revista de Filología y Lingüística de la Universidad de Costa Rica, vol. XI (1): 119 - 130.

Francis, W. N. 1983. Dialectology. An introduction. Londres y Nueva York: Longman Group Limited.

Gagini, Carlos. 1892. Diccionario de barbarismos y provincialismos de Costa Rica. San José: Tipografía Nacional.

1918. Diccionario de costarriqueñismos. San José: Imprenta Nacional.

Gellner, Ernest. 1959. Words and Things. Londres: Victor Gollancz Ltd.

Goebl, H. (ed.). 1984. Dialectology. Bochum: Studienverlag Dr. N. Brockmeyer.

Gómez Córdoba, C. E. 1975. Vocabulario cafetero de la provincia de Cartago. Tesis de Licenciatura: Universidad de Costa Rica.

González Álvarez, L. et al. 1993. Comunidades pesquero - artesanales en Costa Rica. Heredia: Editorial de la Universidad Nacional.

Guitart, Jorge M. y Joaquín Roy (eds.). 1980. La estructura fónica de la lengua castellana: fonología, morfología, dialectología. Barcelona: Editorial Anagrama.

Guitart, Jorge M. y Juan C. Zamora Munné. 1988. Dialectología hispanoamericana. Teoría, descripción, historia. Salamanca: Ediciones Almar. 
Gulden, C. M. van der. 1994. Vocabulario nicaragüense. Managua: Editorial UCA. Colección Alternativa. Serie Habla Nicaragüense $N^{\circ} 1$.

Haber H. (ed.). 1994. Costa Rica. Insight Guides. APA Publications Ltd.

Hernández, C. et al. 1991. El español de América. Actas del III Congreso Internacional del Español de América. Valladolid, 3 a 9 de julio de 1989. Tres volúmenes. Salamanca: Junta de Castilla y León.

Herzfeld, Anita. 1978. "Vida o muerte del criollo limonense.” Revista de Filología y Lingüística de la Universidad de Costa Rica, vol. IV (2).

1994. "Language and Identity: The Black Minority of Costa Rica." Revista de Filología y Lingüística de la Universidad de Costa Rica. XX (1): 113 - 142.

Jaberg, K. y J. Jud. 1931. Sprach- und Sachatlas des Italiens und der Südschweiz. 2 volúmenes. Zofingen: Verlagsanstalt Ringier \& Co.

Kirkpatrick, B. (comp.). 1995. The Concise Oxford Thesaurus. A Dictionary of Synonyms: Londres - Nueva York - Sydney - Toronto: BCA.

Kunnskapsforlaget. 1989. Store norske leksikon. 15 tomos. Oslo: Aschehoug \& Gyldendals forlag.

Le Guern, Michel. 1990. La metáfora y la metonimia. 5.a ed. Madrid: Ediciones Cátedra, S.A.

Lope Blanch, J. M. 1975. "Delimitación de las zonas dialectales de México: objetivos y problemas." Hispania 58.

1989. Estudios de lingüística hispanoamericana. México: Universidad Nacional Autónoma de México.

1990. Atlas lingüístico de México. México: El Colegio de México, Fondo de Cultura Económica.

Louis, Betzy Bell. 1997. El léxico del cuerpo humano en Costa Rica: Análisis dialectológico - semántico. Tesina de Hovedfag: Universidad de Bergen.

Luzio, Aldo di y Peter Auer (eds.). 1988. Variation and Convergence. Studies in Social Dialectology. Berlín, Nueva York: Walter de Gruyter.

Lyons, John. 1991. Semantics: 1. Cambridge: Cambridge University Press. 
Masís Morales, Oscar y Elizabeth Mora Lobo. 1985. "Las oraciones completivas en el criollo de Limón: Análisis sintáctico de un idiolecto". Revista de Filología y Lingüística de la Universidad de Costa Rica. XI (2): 111-121.

Mc Arthur, T. 1987. Longman Lexicon of Contemporary English. Londres: Longman Group UK Limited.

Meléndez, C. y Q. Duncan. 1993. El negro en Costa Rica. 10. ed. San José: Editorial Costa Rica.

Meza, Hortensia. 1980. El habla de los sabaneros liberianos y tilaranenses. Estudio léxico - semántico con anotaciones fonológicas, morfológicas y sintácticas. Tesis de Licenciatura: Universidad de Costa Rica.

Moliner, María. 1994. Diccionario de uso del español. Dos tomos. Madrid: Gredos.

Montes Giraldo, José Joaquín. 1970. Dialectología y Geografía Lingüística: Notas de Orientación. Bogotá: Publicaciones del Instituto Caro y Cuervo.

1973. Muestra del léxico de la pesca en Colombia. Bogotá: Publicaciones del Instituto Caro y Cuervo.

1982. Dialectología general e hispanoamericana. Orientación teórica, metodología y bibliografía. Bogotá: Instituto Caro y Cuervo.

1983b. "Habla, lengua e idioma". Thesaurus. XXXVIII: 325-339.

1993a: Motivación y creación léxica en el español de Colombia. Bogotá: Publicaciones del Instituto Caro y Cuervo.

Moreno Fernández, F. 1990. Metodología sociolingüística. Madrid: Editorial Gredos.

Muñiz Rodríguez, V. 1989. Introducción a la filosofía del lenguaje. Problemas ontológicos. Barcelona: Anthropos. Editorial del Hombre.

Navarro, T. 1948/1974. El español en Puerto Rico. Universidad de Puerto Rico: Editorial Universitaria.

Petyt, K. M. 1980. The Study of Dialect. An introduction to dialectology. Londres: The Language Library. André Deutsch Ltd.

Portilla Chaves, Mario. 1993. "Fonemas segmentales en el criollo inglés de Limón.” En: Revista de Filología y Lingüística de la Universidad de Costa Rica. XIX (2): 89 - 97. 
Quesada Pacheco, Miguel Ángel. 1981. Análisis sociológico del español de San Gabriel, Monterrey y la Legua de Aserrí. Tesis de Licenciatura: Universidad de Costa Rica.

1985. Diccionario regional de los distritos de San Gabriel, Monterrey y la Legua de Aserrí. San Gabriel de Aserrí: Centro de Producciones Audiovisuales.

1991. El español de Guanacaste. San José: Editorial de la Universidad de Costa Rica.

1992a. El español en Costa Rica. San José: Editorial Fernández - Arce.

1992b. "Pequeño atlas lingüístico de Costa Rica". Revista de Filología y Lingüística de la Universidad de Costa Rica, XVIII (2): 85-190.

1992c. Atlas Lingüístico - etnográfico de Costa Rica. Cuestionario. San José: Editorial Nueva Década.

1996. Nuevo diccionario de costarriqueñismos. Segunda ed. Cartago: Editorial Tecnológica de Costa Rica.

Real Academia Española. 1996. Diccionario de la lengua española. 21. edición. Madrid: Espasa Calpe.

Revilla, Ángel. 1976. Panameñismos. Panamá: Roysa.

Román, J. M. 1976. El uso del español en Desamparados de Alajuela. Tesis de Licenciatura: Universidad de Costa Rica.

Roy, Joaquín y Jorge M. Guitart (eds.). 1980. La Estructura fónica de la lengua castellana Fonología, morfología, dialectología. Barcelona: Editorial Anagrama.

Sánchez, V. 1983. Cuestionario lingüístico costarricense. San José: Editorial de la Universidad de Costa Rica.

Smith et al. 1987. Collins Spanish - English English - Spanish Dictionary. Londres - Glasgow: Collins.

The Oxford Guide to the English Language. 1983. Londres: Guild Publishing: 1981

Thomas, Alan R.(ed.). 1988. Methods in Dialectology. Clevedon, Philadelphia: Multilingual Matters LTD.

Thompson, D. (ed.). 1995. The Concise Oxford Dictionary. Ninth edition. BCA, Londres Nueva York - Sydney - Toronto: BCA. 
Thun, Harald. 1990. Atlas lingüístico diatópico - diastrático del Uruguay. Bamberg - Stuttgart: Franz Steiner Verlag.

Vindas Chaves, F. 1971. Vocabulario del banano. Tesis de Licenciatura: Universidad de Costa Rica.

VOX. 1988. Diccionario manual ilustrado de la lengua española. Barcelona: Bibliograf S/A.

Wenker, G. 1881. Sprachatlas des Deutschen Reiches. Marburgo - Berlin.

Wilson, J. 1970. A Generative Phonological Study of Costa Rican Spanish. Tesis doctoral: Universidad de Michigan.

Wrede, F. et al. 1926. Deutscher Sprachatlas. Marburg: Elwert.

Wright, Fernando M. 1975. "Un análisis sintáctico del habla criolla de Limón”. Revista de Filología y Lingüística de la Universidad de Costa Rica. Vol. I (2).

Wright, Fernando M. 1982. "Problemas y métodos para la enseñanza del inglés como segunda lengua a los hablantes del Mek-a-tel-yu en la provincia de Limón”. Revista de Filología y Lingüística de la Universidad de Costa Rica, VIII (1 y 2).

Zgusta Ladislav (ed.). 1980. Theory and Method in Lexicography: Western and Non - Western Perspectives. South Carolina - Columbia: Hornbeam Press, Incorporated. 
152

Filología y Lingüística XXXI (2): 119-156, 2005 / ISSN: 0377-628X

Anexo: Figuras
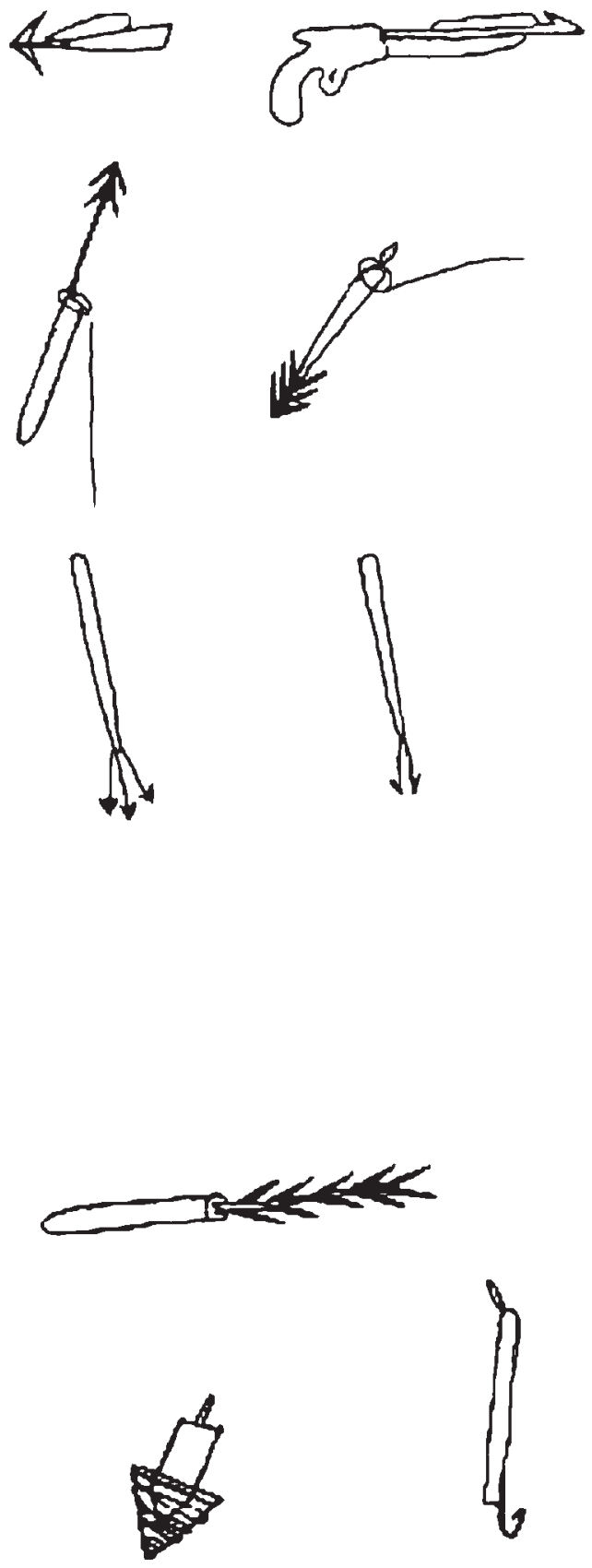

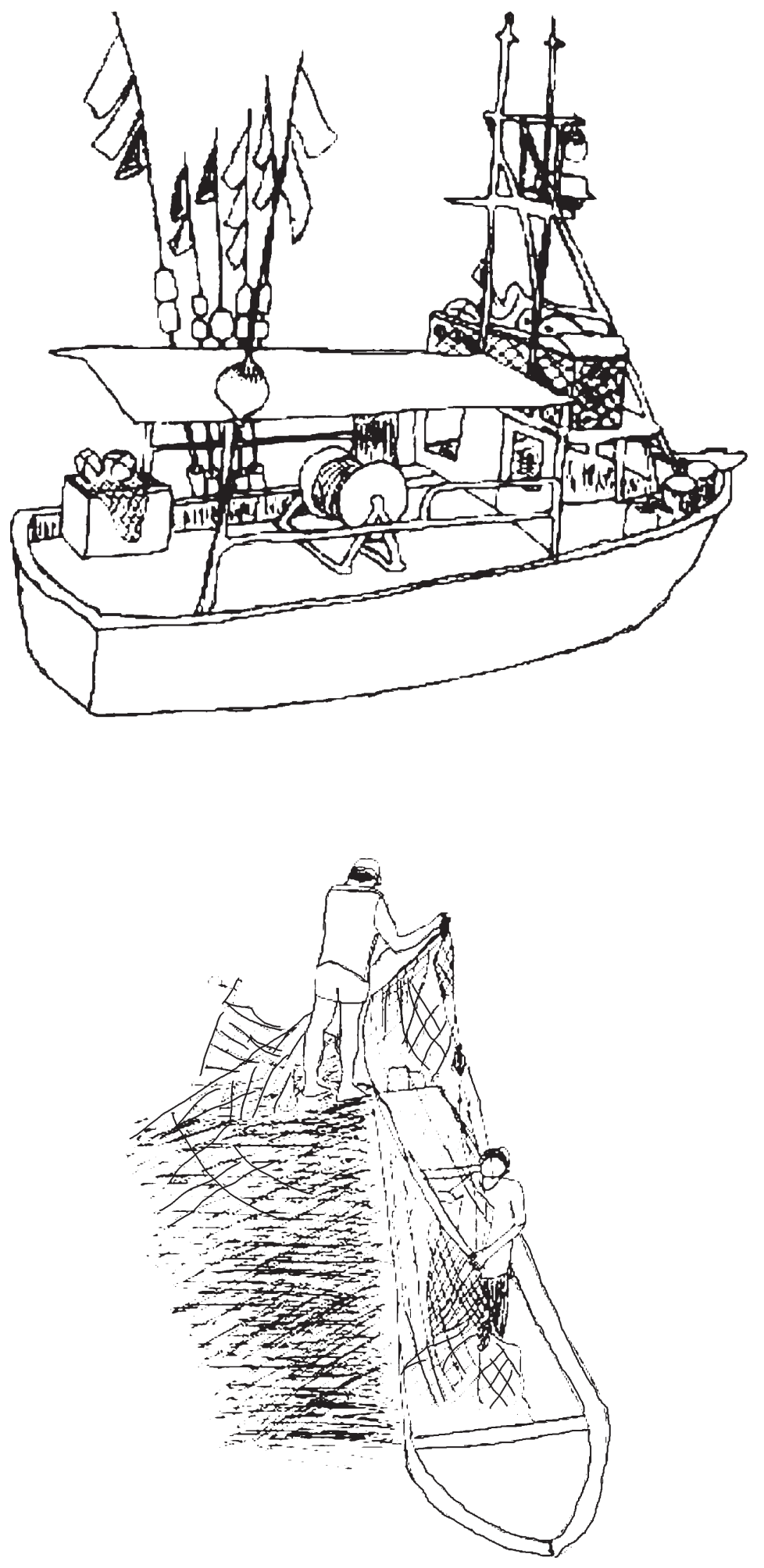

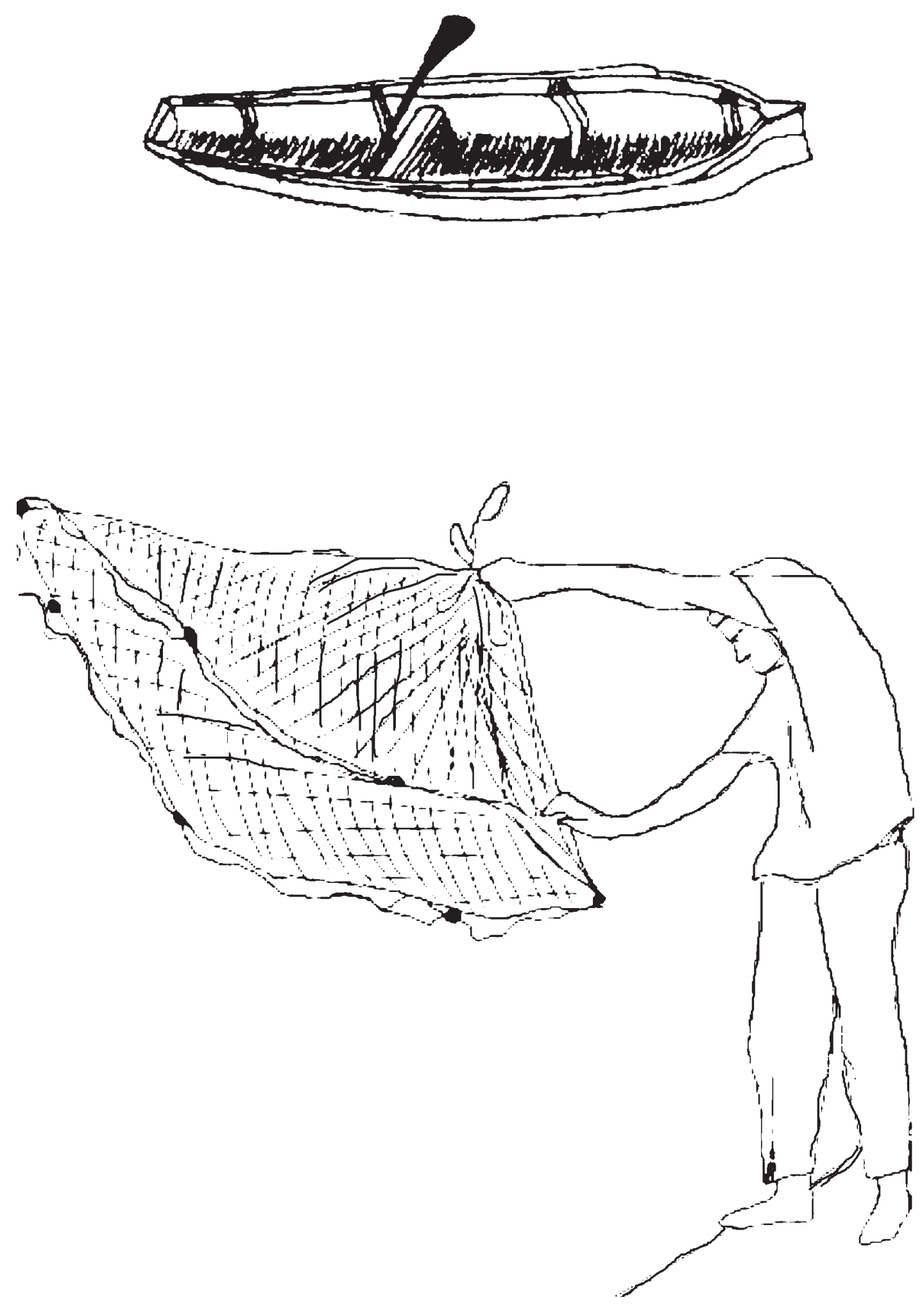

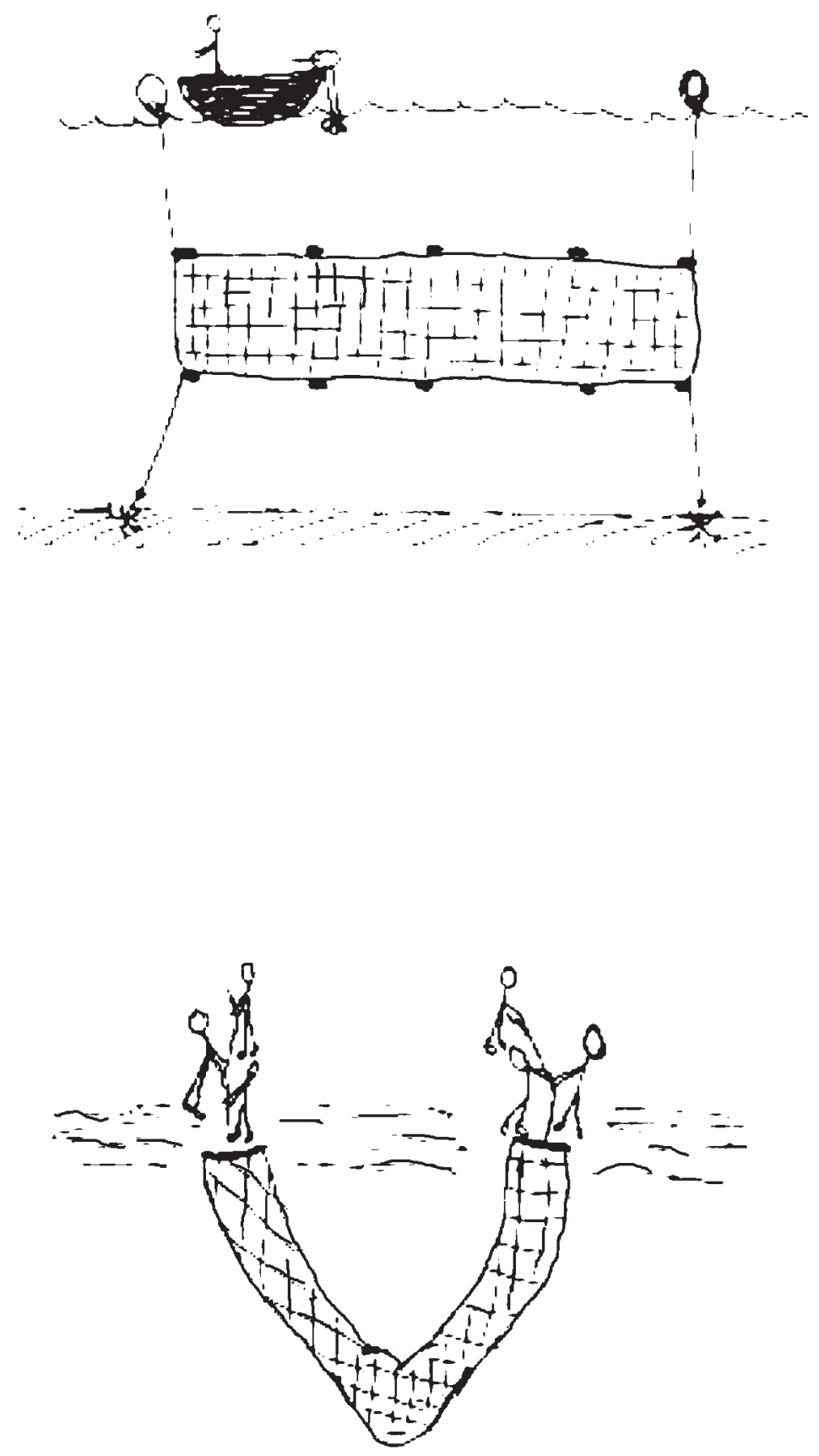

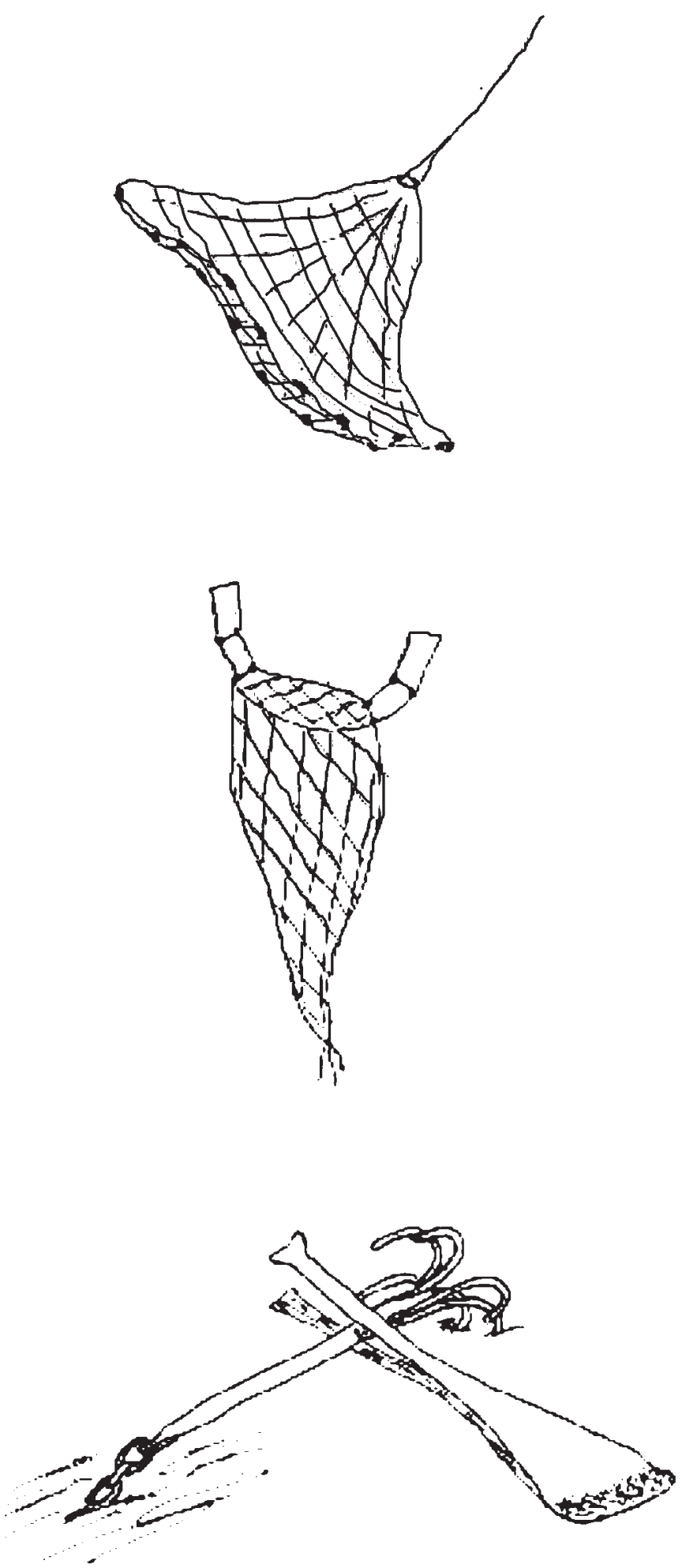